\title{
Efficacy of vitamin D plus calcium with/without alendronate on bone metabolism in immunologic thrombocytopenic purpura patients with steroid treatment: Nine-month results of a randomized, double-blinded, controlled trial
}

\author{
XIA LIN ${ }^{1}$, HUI-QI ZHANG ${ }^{1}$, LI-HONG SHOU ${ }^{2}$, XIANG-LI SHEN ${ }^{1}$ and ZONG-XIN ZHANG ${ }^{3}$ \\ ${ }^{1}$ Department of Hematology, The First People's Hospital of Huzhou, \\ The First Affiliated Hospital of Huzhou University, Huzhou, Zhejiang 313000; ${ }^{2}$ Department of Hematology; \\ ${ }^{3}$ Clinical Laboratory, Huzhou Central Hospital, Huzhou, Zhejiang 313003, P.R. China
}

Received July 22, 2018; Accepted December 31, 2018

DOI: $10.3892 /$ etm.2019.7694

\begin{abstract}
Bone loss is a prominent complication in immunologic thrombocytopenic purpura (ITP) patients with steroid treatment. Anti-osteoporotic medications are applied as a therapeutic strategy to prevent bone deterioration in ITP patients. However, the skeletal protective effect of alendronate (ALN) in ITP patients has been rarely reported. The present study was performed to determine whether ALN reduces bone loss in ITP patients. A total of 40 ITP patients with steroid treatment were randomized into a placebo group $[n=20$; caltrate D (CalD)] and an ALN (10 mg/day) + CalD group $(n=20)$. The patients received CalD or CalD + ALN treatment for 9 months. The primary outcomes were bone mineral density (BMD) in the lumbar vertebrae (L1-L4), femoral neck and total hip, as well as bone metabolism markers. The results indicated that the BMD of the lumbar vertebrae (L1-L4), femoral neck and total hip was significantly increased after ALN + CalD treatment for at 6 and 9 months compared with the baseline. Compared with CalD treatment alone, CalD combined with ALN significantly elevated the BMD at the three skeletal sites at 9 months. Compared with the baseline levels or CalD treatment alone, ALN together with CalD treatment markedly reduced urinary $\mathrm{Ca}$ excretion and the serum levels of the bone resorption markers tartrate resistant acid phosphatase $5 \mathrm{~b}$ and $\mathrm{C}$-terminal telopeptides of type 1 collagen, at 9 months. In conclusion, treatment with ALN together with CalD significantly elevated the BMD at three skeletal sites,
\end{abstract}

Correspondence to: Dr Zong-Xin Zhang, Clinical Laboratory, Huzhou Central Hospital, 198 Hongqi Road, Huzhou, Zhejiang 313003, P.R. China

E-mail: zhang_zx0909@163.com

Key words: immunologic thrombocytopenic purpura, alendronate, steroids, glucocorticoid, osteoporosis and inhibited urinary $\mathrm{Ca}$ excretion and the activity of bone resorption markers in patients with ITP.

\section{Introduction}

Steroids are widely used to inhibit inflammation in a variety of immune-mediated diseases, including systemic lupus erythematosus and arthritis (1-3). However, continuous oral steroid therapy for 3-6 months or longer is associated with bone loss and fractures as the major adverse events (4-6). The fracture risk is positively correlated with the daily and cumulative glucocorticoid dose in patients with glucocorticoid-induced osteoporosis (GIOP) (6-8). A clinical survey indicated that calcium plus vitamin D supplementation has a limited effect on the treatment of GIOP, while specific anti-osteoporotic medicines, including bisphosphonates, alendronate (ALN) and teriparatide, are effective for the management of GIOP (6).

Immunologic thrombocytopenic purpura (ITP) is a hematologic disorder characterized by accelerated platelet consumption, shortened platelet survival and impaired platelet production (9). In the last two decades, splenectomy, rituximab and thrombopoietin-receptor agonists have been introduced for the treatment of patients with ITP (3). Furthermore, corticosteroids are considered as the first-line and mainstay of treatment and are recommended by the American Society of Hematology and the international consensus report on the investigation and management of primary ITP $(10,11)$. For instance, prednisone at $1 \mathrm{mg}$ /day for 3-4 weeks followed by tapering for another 2-3 weeks is a general standard approach for ITP treatment (3). Unfortunately, a glucocorticoid-induced decrease in bone mineral density (BMD) is observed in patients with ITP (12). Therefore, ALN was co-administered to prevent bone loss in patients with ITP in the present study.

Previous studies have validated that treatment with ALN reduces bone loss and the risk of fracture in patients with GIOP (13), normocalcemic primary hyperparathyroidism (14), ankylosing spondylitis (15), orthotopic liver transplantation (16) and post-menopausal osteoporosis (17). The present study aimed to evaluate the efficacy and safety of ALN (70 mg) 
weekly combined with caltrate D (CalD; Ca, 1,200 mg and vitamin $\mathrm{D}_{3}, 250 \mathrm{IU}$ ) daily to prevent bone loss in comparison to CalD treatment alone in ITP patients under glucocorticoid treatment. The BMD at the lumbar vertebrae (L1-L4), femoral neck and total hip, as well as bone metabolic parameters were assessed in patients with ITP. At the 9-month follow up, the results demonstrated that ALN prevented urinary $\mathrm{Ca}$ excretion and loss in BMD, and reduced bone resorption markers in patients with ITP.

\section{Materials and methods}

Patients. A total of 105 ITP patients were recruited from the First People's Hospital of Huzhou (Huzhou, China) and Huzhou Central Hospital (Huzhou, China) between January 2014 and June 2017. The patients were between 18 and 50 years of age and provided written informed consent to participate in the study. The inclusion and exclusion criteria are presented in Fig. 1. Among the candidate ITP patients, 7 post-menopausal women were excluded due to their clinical presentation, pelvioscopy results and serum levels of follicle-stimulating hormone $(>10 \mathrm{U} / \mathrm{l})$ and estradiol $(<20 \mathrm{pg} / \mathrm{ml})$. The baseline physiological and biochemical parameters of all patients are provided in Tables I and II. The effect of steroids on bone loss in ITP patients mainly depends on the accumulated dose. Before the commencement of the present trial, all ITP patients received steroid therapy for one month. At 1 month prior to the start of the trial, the steroid treatment was discontinued for all ITP patients, observation or replacement therapy with gamma globulin.

Patient randomization was performed using the SAS 9.0 system (SAS Institute, Cary, NC, USA). The 40 enrolled ITP patients were randomized into CalD or CalD + ALN treatment groups at a pre-determined proportion of 1:1. The randomization process was an unsupervised classification. Before the start of the trial, the participants were given a fixed prescription and received study medications from pharmacy staff according to the enrollment sequence. The medication for the CalD and CalD + ALN groups had the same appearance, shape, color and packaging, so that the research physicians and participants were not distinguish them. The research physicians and participants were not informed about any individual treatment details until the end of the treatment course and after receiving the laboratory test results. The Huzhou Central Hospital (Huzhou, China) approved the study protocol (approval no. 201401A006). Informed consent forms were signed by all patients. None of the patients were included in the current study prior to obtaining ethical approval.

Treatment. The patients in the CalD group $(n=20)$ received 1,200 mg calcium and 250 IU vitamin $\mathrm{D}_{3}$ daily (Caltrate D; Pfizer, Inc., New York, NY, USA). Patients in the CalD + ALN group $(n=20)$ received $1,200 \mathrm{mg}$ calcium and $250 \mathrm{IU}$ vitamin $\mathrm{D}_{3}$ daily with ALN (70 mg; Merck \& Co., Darmstadt, Germany) weekly for 9 months.

Measurement of BMD. The BMD from the first to fourth lumbar vertebrae (L1-L4), femoral neck and total hip were measured by dual-energy X-ray absorptiometry (Hologic DQR-4500W; Hologic, Inc., Bedford, MA, USA), according to the manufacturer's protocol (16). Osteoporosis and osteopenia were defined according to international guidelines: Osteoporosis was classified by a t-score of <-2.5 standard deviation (SD) in either lumbar spine or femur, and osteopenia by a t-score between -2.5 and -1 SD (18).

Bone metabolism markers. Serum and urine samples for determination of $\mathrm{Ca}$ and $\mathrm{P}$ were obtained at baseline levels and after 1, 3, 6 and 9 months, using an Olympus AU 2700 automated multichannel analyzer (Olympus, Tokyo, Japan). Bone formation and resorption markers in the serum were determined at the baseline and after 1, 3, 6 and 9 months. Serum intact parathyroid hormone (PTH; cat. no. 11972219122) and osteocalcin (cat. no. 12149133122) were measured using chemiluminescence immunoassays (Roche Diagnostics, Mannheim, Germany). Serum $25(\mathrm{OH})$ vitamin D was determined using a DiaSorin kit on a LIAISON automated immunoassay analyzer (DiaSorin, Saluggia, Italy). Serum bone-specific alkaline phosphatase (cat. no. 48262; BAP; Access Ostase, Beckman Coulter, Inc., Brea, CA, USA), type 1 procollagen $\mathrm{N}$-terminal propeptide (cat. no. E-EL-H0185c; P1NP; Elabscience Biotechnology Co., Ltd, Wuhan, China), tartrate resistant acid phosphatase $5 \mathrm{~b}$ (cat. no. CSB-E08490h; TRACP-5b; CUSABIO Technology LCC, Wuhan, Hubei, China) and C-terminal telopeptides of type 1 collagen (cat. no. E-EL-H0960c; CTX; Elabscience Biotechnology Co., Ltd, Wuhan, China) were measured with a commercial ELISA. All tests were performed according to the manufacturers' instructions in a routine laboratory.

Statistical analysis. Continuous data are expressed as the mean \pm standard deviation. A two-tailed, unpaired Student's $\mathrm{t}$-test for independent samples or an $\chi^{2}$ test was used to compare baseline variables between the two groups. The differences between quantitative variables were analyzed using a Student's t-test for unpaired data. Inter-group differences were analyzed by one-way analysis of variance, followed by Tukey's post-hoc test. SPSS (v.17; SPSS, Inc., Chicago, IL, USA) for Windows was used for statistical analysis and GraphPad Prism software (version 7.0; GraphPad Software, Inc., La Jolla, CA, USA) was used to draw the histograms. $\mathrm{P}<0.05$ was considered to indicate statistically significant differences.

\section{Results}

Study population and baseline characteristics. Initially, 105 patients with a clinical diagnosis of ITP and who had received steroids treatment were recruited for the present study. Among these patients, 67 were included based on the following: i) An age of 18-50 years; ii) no use of steroids, calcium, vitamin D, anti-inflammatory agents or anti-osteoporotic drugs within one month prior to the trial; iii) the patients provided written informed consent form. In addition, 27 patients were excluded due to co-morbidities or post-menopausal status, and the remaining 40 patients were randomized into two groups (20 in the ALN + CalD group and 20 in the CalD group; Fig. 1).

The baseline clinicopathological characteristics and biochemical parameters of the patients with ITP are presented in Tables I and II, respectively. At the baseline, clinicopathological characteristics, including the gender ratio, age, BMI, 


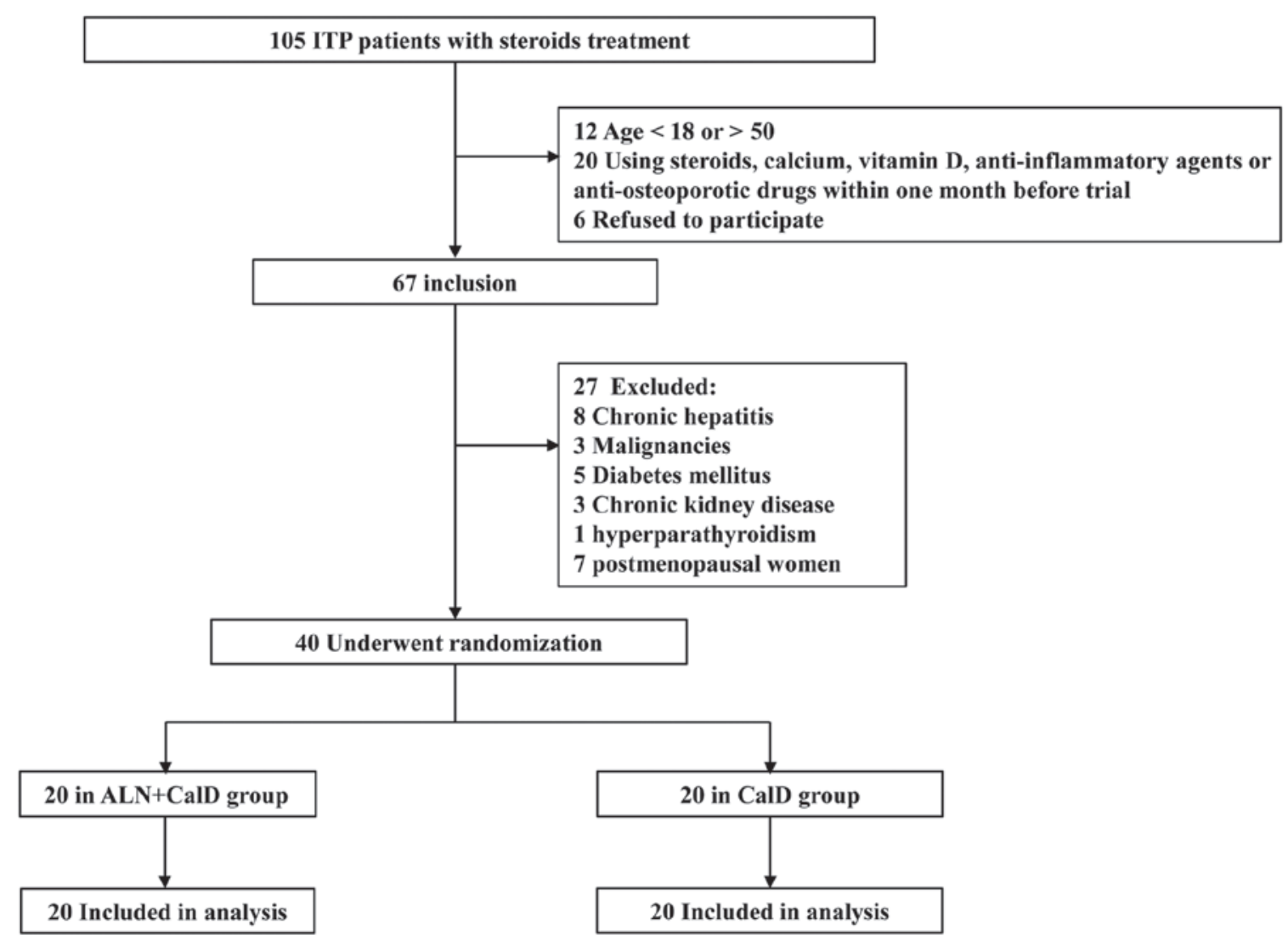

Figure 1. Flowchart of the study. ITP, immunologic thrombocytopenic purpura; ALN, alendronate; CalD, caltrate D.

steroid dosage and therapy duration, platelet count, as well as the BMD of lumbar vertebrae, femoral neck and total hip, were not significantly different between the ALN + CalD and CalD groups $(\mathrm{P}>0.05$; Table I). Furthermore, the baseline levels of serum Ca, P, intact PTH, 25(OH) vitamin D, BAP, P1NP, osteocalcin, TRACP-5b, CTX and 24-h urinary Ca were not significantly different between the two groups (Table II).

Outcomes. First, the association between BMD (T-score) and the average daily steroid dose was demonstrated in patients with ITP. The BMD T-score was $-0.64 \pm 0.28$ in the group receiving $>5 \mathrm{mg} /$ day, $-1.54 \pm 0.48$ in the group treated with $5-10 \mathrm{mg} /$ day, $-2.74 \pm 0.30$ in the group receiving $10-15 \mathrm{mg} /$ day and $-3.34 \pm 0.66$ in the group receiving $>15 \mathrm{mg} /$ day, suggesting that average daily steroid dose was positively correlated with bone loss in ITP patients with steroids treatment prior to clinical trial (Fig. 2).

The BMD of the lumbar vertebrae L1-L4 was significantly increased between month 3 and 9 of ALN + CalD treatment compared with that at the baseline. Compared with the baseline levels, CalD treatment alone also increased the BMD of the lumbar vertebrae L1-L4 at month 9 in patients with ITP. These results suggest that ALN + CalD and CalD have a beneficial effect to improve the BMD of the lumbar vertebrae L1-L4 in patients with ITP. Of note, the BMD of the lumbar vertebrae L1-L4 in the ALN + CalD group was significantly higher than that in the CalD group at month 9 ( $\mathrm{P}<0.05$; Table III; Fig. 3A). In addition, the BMD of the femoral neck and total hip was significantly increased in the ALN + CalD group compared with that at the baseline at month 6 and 9. However, CalD treatment alone had no significant effect on the BMD of the femoral neck and total hip in patients with ITP. Of note, ALN combined with CalD markedly increased the BMD of the femoral neck and total hip as compared with that in the CalD group at month 9 ( $\mathrm{P}<0.05$; Table III; Fig. 3B and C). These results indicate that ALN combined with CalD is superior to CalD treatment alone in protecting against steroid-associated bone deterioration in patients with ITP.

No change of serum $\mathrm{Ca}$ from the baseline was observed at each time-point in each of the two treatment groups (Fig. 4A). There was no difference in serum $\mathrm{Ca}$ between the CalD and $\mathrm{ALN}+\mathrm{CalD}$ groups, except at month 1 . Of note, after 9 months of ALN + CalD treatment, the 24-h urinary Ca excretion was significantly suppressed compared with the baseline levels. Compared with CalD treatment alone, ALN + CalD caused a significant decrease in 24-h urinary $\mathrm{Ca}$ at 9 months. However, CalD treatment alone did not significantly change the 24-h urinary $\mathrm{Ca}$ at any time-point (Fig. 4B). Compared with the baseline levels or CalD treatment alone, ALN + CalD treatment markedly decreased serum $\mathrm{P}$ at month 1 and 9. CalD treatment alone had on significant effect on serum $\mathrm{P}$ at any time-point (Fig. 4C). Neither CalD treatment alone nor CalD combined with ALN had any effect on PTH at any time-point (Fig. 4D). An increase of $25(\mathrm{OH})$ vitamin D levels was detected in the CalD and CalD combined with ALN groups after 3 months of treatment; however, no significant difference in $25(\mathrm{OH})$ vitamin $\mathrm{D}$ levels was observed between the two groups (Fig. 4E).

The present study also indicated that CalD treatment alone or combined with ALN provided no significant improvement in the 
Table I. Baseline clinicopathological characteristics of patients with immunologic thrombocytopenic purpura.

\begin{tabular}{|c|c|c|c|}
\hline Parameter & ALN+CalD $(n=20)$ & CalD $(n=20)$ & P-value \\
\hline Gender (M/F) & $15 / 5$ & $13 / 7$ & 0.490 \\
\hline Age (years) & $37.6 \pm 9.2$ & $35.5 \pm 8.1$ & 0.449 \\
\hline BMI $\left(\mathrm{kg} / \mathrm{m}^{2}\right)$ & $23.7 \pm 2.2$ & $22.8 \pm 2.2$ & 0.215 \\
\hline Steroid dosage (mg/day) & $13.15 \pm 6.70$ & $12.15 \pm 5.86$ & 0.619 \\
\hline Steroid therapy duration (days) & $479 \pm 187$ & $505 \pm 177$ & 0.646 \\
\hline Platelet count $\left(\times 10^{4} / \mathrm{mm}^{3}\right)$ & $13.7 \pm 4.3$ & $14.2 \pm 3.9$ & 0.673 \\
\hline Bone status $(\%)$ & & & 0.609 \\
\hline Normal (T-score >-1 SD) & $4(20)$ & $5(25)$ & \\
\hline Osteopenia $(-2.5 \mathrm{SD}<\mathrm{T}$-score $\leq-1 \mathrm{SD})$ & $6(30)$ & $7(35)$ & \\
\hline Osteoporosis (T-score $\leq-2.5 \mathrm{SD}$ ) & $10(50)$ & $8(40)$ & \\
\hline \multicolumn{4}{|l|}{ Bone mineral density $\left(\mathrm{g} / \mathrm{cm}^{2}\right)$} \\
\hline Lumbar L1-L4 & $0.816 \pm 0.052$ & $0.832 \pm 0.061$ & 0.375 \\
\hline Femoral neck & $0.752 \pm 0.059$ & $0.741 \pm 0.065$ & 0.592 \\
\hline Total hip & $0.656 \pm 0.067$ & $0.670 \pm 0.054$ & 0.461 \\
\hline
\end{tabular}

Values are expressed as $\mathrm{n}(\%)$ or the mean \pm standard deviation. ALN, alendronate; CalD, caltrate D; M, male; F, female; BMI, body mass index; SD, standard deviation.

Table II. Baseline biochemical parameters of patients with ITP.

\begin{tabular}{lccc}
\hline Parameter & ALN+CalD (n=20) & CalD (n=20) & P-value \\
\hline Serum Ca (mg/dl) & $10.21 \pm 0.58$ & $9.79 \pm 0.71$ & 0.517 \\
24-h urinary Ca (mg/day) & $215 \pm 137$ & $184 \pm 91$ & 0.438 \\
Serum P (mg/dl) & $4.17 \pm 0.56$ & $4.28 \pm 0.48$ & 0.715 \\
Serum intact-PTH (pg/ml) & $46.1 \pm 18.4$ & $50.9 \pm 20.5$ & 0.563 \\
25(OH) vitamin D (ng/ml) & $19.5 \pm 8.3$ & $21.4 \pm 9.2$ & 0.279 \\
BAP (ng/ml) & $21.6 \pm 8.2$ & $18.5 \pm 9.7$ & 0.288 \\
P1NP (ng/ml) & $53.9 \pm 21.7$ & $60.3 \pm 24.4$ & 0.189 \\
Osteocalcin (ng/ml) & $31.6 \pm 5.9$ & $28.7 \pm 6.5$ & 0.419 \\
TRACP-5b (U/l) & $567 \pm 173$ & $515 \pm 164$ & 0.663 \\
Serum CTX (ng/ml) & $0.457 \pm 0.163$ & $0.406 \pm 0.154$ \\
\hline
\end{tabular}

ITP, immunologic thrombocytopenic purpura; ALN, alendronate; CalD, caltrate D; Ca, calcium; P, phosphorus; PTH, parathyroid hormone; BAP, bone-specific alkaline phosphatase; P1NP, type 1 procollagen $\mathrm{N}$-terminal propeptide; TRACP-5b, tartrate resistant acid phosphatase 5b; CTX, C-terminal telopeptides of type 1 collagen.

bone formation markers BAP, P1NP and osteocalcin (Fig. 5A, B and $\mathrm{C}$, respectively), while the bone absorption markers TRACP-5b and CTX were inhibited by CalD combined with ALN treatment (Fig. 5D and E). Compared with the baseline levels, ALN + CalD treatment resulted in a significant decrease in TRACP-5b levels after 3 months of treatment. TRACP-5b was significantly lower in the ALN + CalD group compared with that in the CalD group at month 6 and 9 (Fig. 5D). Compared with the baseline levels, ALN + CalD treatment led to a reduction of CTX levels in the serum after 9 months of treatment. The levels of CTX were significantly decreased in the ALN + CalD group compared with those in the CalD group. These results suggested that ALN had a specific effect to inhibit bone resorption markers in patients with ITP.

\section{Discussion}

Emerging evidence has indicated that ITP patients commonly present with osteopenia or osteoporosis, which may be attributed to a prolonged use of steroid drugs in numerous refractory cases $(12,19)$. Nomura et al $(12)$ reported that administration of bisphosphonate, a novel anti-metabolic osteopathy drug, increased the BMD of lumbar vertebrae and decreased urinary levels of collagen type 1 cross-linked $\mathrm{N}$-telopeptides, a bone resorption marker. In the present study, ALN (70 mg), an anti-osteoporosis medication, combined with CalD (Ca, 1,200 mg and vitamin $\mathrm{D}_{3}$, $250 \mathrm{IU}$ ) was demonstrated to increase the BMD at the lumbar vertebrae (L1-L4), femoral neck and total hip, inhibit urinary Ca excretion and 
Table III. BMD of lumbar, femoral neck and hip in the two patient groups at different time-points within the study period.

\begin{tabular}{lccccl}
\hline Site/group & Baseline & 1 month & 3 months & 6 months & 9 months \\
\hline Lumbar L1-L4 $\left(\mathrm{g} / \mathrm{cm}^{2}\right)$ & & & & & \\
ALN+CalD & $0.816 \pm 0.052$ & $0.845 \pm 0.057$ & $0.857 \pm 0.063^{\mathrm{a}}$ & $0.871 \pm 0.069^{\mathrm{b}}$ & $0.922 \pm 0.073^{\mathrm{c}, \mathrm{d}}$ \\
CalD & $0.832 \pm 0.061$ & $0.841 \pm 0.051$ & $0.849 \pm 0.060$ & $0.855 \pm 0.064$ & $0.873 \pm 0.065^{\mathrm{a}}$ \\
Femoral neck $\left(\mathrm{g} / \mathrm{cm}^{2}\right)$ & & & & \\
ALN+CalD & $0.752 \pm 0.059$ & $0.761 \pm 0.054$ & $0.783 \pm 0.060$ & $0.799 \pm 0.062^{\mathrm{a}}$ & $0.811 \pm 0.065^{\mathrm{b}, \mathrm{d}}$ \\
CalD & $0.741 \pm 0.065$ & $0.746 \pm 0.061$ & $0.759 \pm 0.055$ & $0.766 \pm 0.060$ & $0.768 \pm 0.064$ \\
Total hip $\left(\mathrm{g} / \mathrm{cm}^{2}\right)$ & & & & & \\
ALN+CalD & $0.656 \pm 0.067$ & $0.671 \pm 0.065$ & $0.693 \pm 0.068$ & $0.722 \pm 0.070^{\mathrm{b}}$ & $0.755 \pm 0.072^{\mathrm{c}, \mathrm{e}}$ \\
CalD & $0.670 \pm 0.054$ & $0.664 \pm 0.050$ & $0.675 \pm 0.055$ & $0.683 \pm 0.057$ & $0.692 \pm 0.060$ \\
\hline
\end{tabular}

BMD, bone mineral density; ALN, alendronate; CalD, caltrate $\mathrm{D} .{ }^{\mathrm{a}} \mathrm{P}<0.05,{ }^{\mathrm{b}} \mathrm{P}<0.01,{ }^{\mathrm{c}} \mathrm{P}<0.001$ compared with baseline; ${ }^{\mathrm{d}} \mathrm{P}<0.05$, ${ }^{\mathrm{e}} \mathrm{P}<0.01$ compared with CalD group at the same time-point.

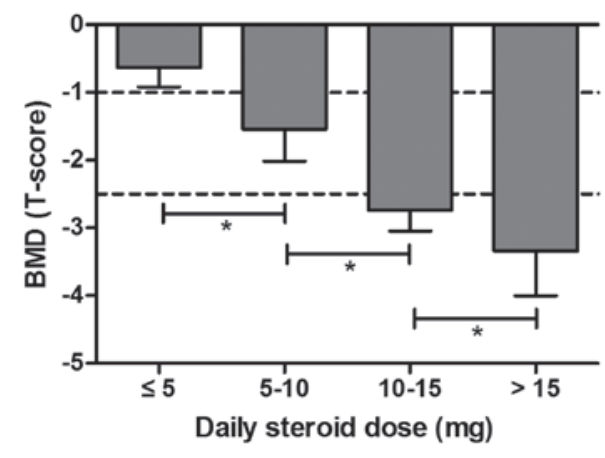

Figure 2. Impact of steroid treatment on BMD in patients with ITP. ${ }^{*} \mathrm{P}<0.05$. BMD, bone mineral density.

the activity of bone resorption markers TRACP-5b and CTX, compared with the baseline. The results also suggested that ALN combined with CalD is superior to CaldD alone in the prevention of bone loss in ITP patients with steroid administration. A significant increase in the BMD of lumbar vertebrae, but not in the femoral neck and total hip, and serum $25(\mathrm{OH})$ vitamin $\mathrm{D}$ levels were identified with CaldD treatment alone for 9 months, compared with the baseline levels.

A number of trials have reported on the beneficial effect of ALN in the treatment of bone deteriorations in post-menopausal women, as well as in GIOP and orthotopic liver transplantation-associated bone loss $(13,16,17,20)$. A comparative study of ALN (10 mg/day), calcitriol or simple vitamin D supplementation in 195 glucocorticoid-treated subjects with a 2-year follow-up reported a significant increase in the mean lumbar vertebral BMD $(+5.9 \%)$ with ALN treatment, compared with that achieved by calcitriol $(-0.7 \%)$ or vitamin D (-0.5\%) treatment (13). A meta-analysis of seven studies comprising 1,111 patients with GIOP indicated that ALN administration markedly elevated the BMD in lumbar vertebrae and femoral neck (21). However, only few randomized controlled trials have addressed the role of ALN in the management of bone loss in patients with ITP. The present study was the first to evaluate the effect of ALN and CalD in patients with ITP. Consistent with previous results, the present
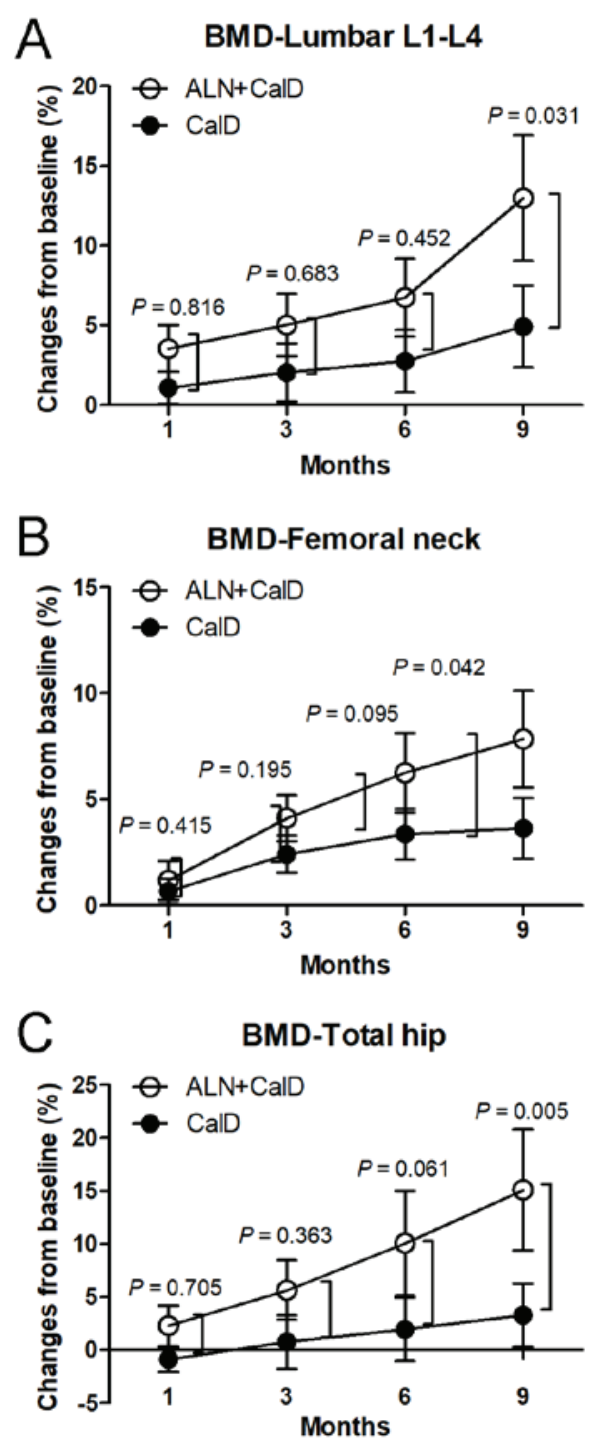

Figure 3. Impact of ALN on the BMD in the lumbar vertebrae, femoral neck and total hip. Percent changes from the baseline of (A) the lumbar vertebrae, (B) femoral neck and (C) total hip in the groups treated with CalD or CalD + ALN for 9 months. BMD, bone mineral density; ALN, alendronate; CalD, caltrate D. 

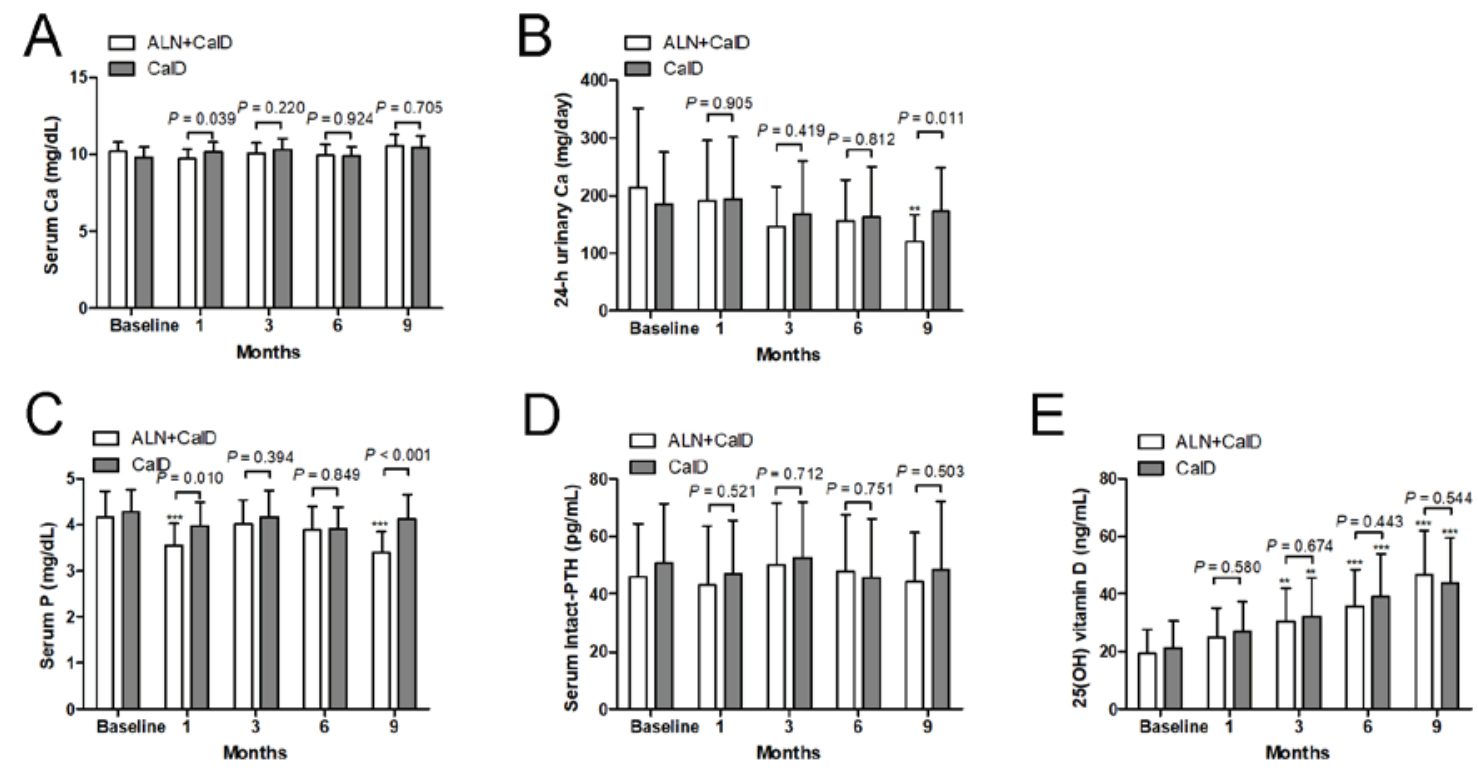

Figure 4. Impact of ALN on Ca and P metabolism in patients with immunologic thrombocytopenic purpura. (A) Serum Ca, (B) $24 \mathrm{~h}$ urinary Ca, (C) serum $\mathrm{P}$, (D) PTH and (E) 25(OH) vitamin D were measured at baseline levels and after 1, 3, 6 and 9 months treatment with CalD or CalD + ALN. ${ }^{* *} \mathrm{P}<0.01$ and ${ }_{* * * *}^{*}<0.001$ vs. corresponding baseline levels. ALN, alendronate; CalD, caltrate D; PTH, parathyroid hormone.
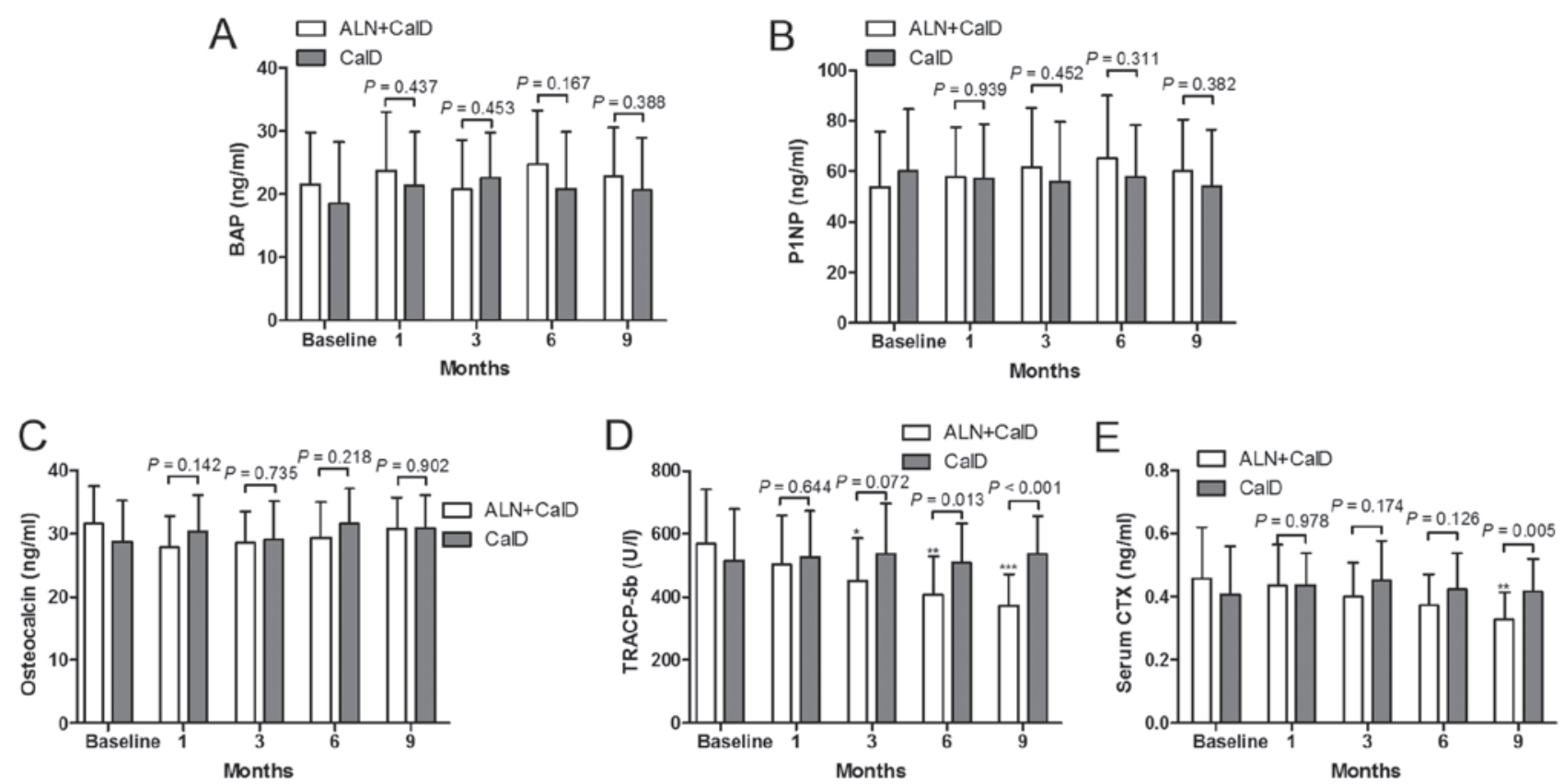

Figure 5. Impact of ALN on bone metabolism markers. (A-C) Bone formation markers (A) BAP, (B) P1NP and (C) osteocalcin, and (D and E) bone resorption markers (D) TRACP-5b and (E) CTX, were measured at baseline levels and after 1,3, 6 and 9 months of treatment with CalD or CalD + ALN. $\mathrm{P}<0.05,{ }^{* *} \mathrm{P}<0.01$ and ${ }^{* * * *} \mathrm{P}<0.001$ vs. baseline levels. ALN, alendronate; CalD, caltrate D; BAP, bone-specific alkaline phosphatase; P1NP, type 1 procollagen N-terminal propeptide; TRACP-5b, tartrate resistant acid phosphatase 5b; CTX, C-terminal telopeptides of type 1 collagen.

study demonstrated the ability of ALN + CalD to increase the BMD of lumbar vertebrae $(+6.74$ and $+12.99 \%)$, femoral neck $(+6.25$ and $+7.84 \%)$ and total hip $(+10.06$ and $+15.09 \%)$ at 6 and 9 months compared with the baseline levels. Compared to CaldD treatment alone, ALN combined with CalD significantly elevated the BMD of the lumbar vertebrae, femoral neck and total hip from +4.93 to $+12.99 \%,+3.64$ to $+7.84 \%$ and +3.28 to $+15.09 \%$, respectively, at 9 months. These results confirmed the beneficial effects of ALN in improving the BMD in GIOP patients.
Numerous studies have indicated that ALN blocks osteoclast differentiation and osteoclastic bone resorption, induces osteoclast precursor apoptosis and inhibits bone resorption markers in vivo and in vitro (22-26). A randomized, controlled trial on ALN in patients with glomerular disease indicated that the serum levels of the bone formation markers PINP and BAP, and the bone resorption marker TRACP-5b, were markedly decreased by ALN treatment for 12 months compared with the baseline levels (27). One previous study in postmenopausal women with 
normocalcemic primary hyperparathyroidism indicated that the bone formation marker osteocalcin and the bone resorption marker CTX were continually reduced over 6 months of ALN treatment (14). A randomized, double-blinded, placebo-controlled trial of ALN treatment for fibrous dysplasia of the bone reported a significant decrease in the bone resorption marker $\mathrm{N}$-terminal telopeptides of type 1 collagen, but no significant effect on serum osteocalcin (28). The present results validated the effects of ALN in increasing the BMD and decreasing bone resorption markers, but no effect on bone formation markers was observed in patients with ITP.

Of note, the present study had certain limitations. The study population was small due to the low prevalence and incidence of ITP. In these patients, the side effects of ALN were not quantitatively evaluated. The bone microstructure was also not evaluated in the ALN-treated ITP patients.

In conclusion, the present study indicated that in ITP patients with steroid treatment, administration of ALN together with CalD over 9 months significantly elevated the BMD at three skeletal sites. Furthermore, treatment with ALN together with CalD markedly inhibited the activity of bone resorption markers. These results suggest that ALN may serve as an effective agent for the prevention and treatment of steroid-induced bone deterioration in patients with ITP scheduled for long-term steroid treatment.

\section{Acknowledgements}

Not applicable.

\section{Funding}

The present study was supported by the Huzhou Science and Technology Plan Program (grant no. 2015GYB28).

\section{Availability of data and materials}

The datasets used and/or analyzed during the present study are available from the corresponding author on reasonable request.

\section{Authors' contributions}

The study was designed by XL and ZZ. Literature research, data acquisition and data analysis were performed by XL, HZ, LS and XS. The manuscript was prepared and edited by XL, HZ, LS, XS and ZZ. Specimen collection and ELISA analysis were performed by XL, HZ, LS and XS. The manuscript was reviewed by XL and ZZ. All authors approved the final version of the manuscript.

\section{Ethical approval and consent to participate}

The Ethics Committee of Huzhou Central Hospital (Huzhou, China) approved the study protocol (approval no. 201401A006). Written informed consent was obtained from all patients.

\section{Patient consent for publication}

All patients provided consent for publication.

\section{Competing interests}

The authors declare that they have no competing interests.

\section{References}

1. Sciascia S, Mompean E, Radin M, Roccatello D and Cuadrado MJ: Rate of adverse effects of medium- to high-dose glucocorticoid therapy in systemic lupus erythematosus: A systematic review of randomized control trials. Clin Drug Investig 37: 519-524, 2017.

2. Safy M, de Hair MJH, Jacobs JWG, Buttgereit F, Kraan MC and van Laar JM: Efficacy and safety of selective glucocorticoid receptor modulators in comparison to glucocorticoids in arthritis, a systematic review. PLoS One 12: e0188810, 2017.

3. Rodeghiero F and Ruggeri M: ITP and international guidelines: What do we know, what do we need? Presse Med 43: e61-e67, 2014.

4. Rentero ML, Amigo E, Chozas N, Fernández Prada M, Silva-Fernández L, Abad Hernandez MA, Rodriguez Barrera JM and del Pino-Montes J; GHDP study group: Prevalence of fractures in women with rheumatoid arthritis and/or systemic lupus erythematosus on chronic glucocorticoid therapy. BMC Musculoskelet Disord 16: 300, 2015.

5. Robinson DE, Dennison EM, Cooper C, van Staa TP and Dixon WG: A review of the methods used to define glucocorticoid exposure and risk attribution when investigating the risk of fracture in a rheumatoid arthritis population. Bone 90: 107-115, 2016.

6. Rizzoli R and Biver E: Glucocorticoid-induced osteoporosis: Who to treat with what agent? Nat Rev Rheumatol 11: 98-109, 2015.

7. Compston J: Glucocorticoid-induced osteoporosis: An update. Endocrine 61: 7-16, 2018.

8. Kanis JA, Johansson $\mathrm{H}$, Oden A, Johnell O, de Laet C, Melton III LJ, Tenenhouse A, Reeve J, Silman AJ, Pols HA, et al: A meta-analysis of prior corticosteroid use and fracture risk. J Bone Miner Res 19: 893-899, 2004.

9. Sugiura T, Yamamoto K, Murakami K, Horita S, Matsusue Y, Nakashima $\mathrm{C}$ and Kirita T: Immune thrombocytopenic purpura detected with oral Hemorrhage: A case report. J Dent (Shiraz) 19: 159-163, 2018.

10. Neunert C, Lim W, Crowther M, Cohen A, Solberg L Jr and Crowther MA; American Society of Hematology: The American Society of Hematology 2011 evidence-based practice guideline for immune thrombocytopenia. Blood 117: 4190-4207, 2011.

11. Provan D, Stasi R, Newland AC, Blanchette VS, Bolton-Maggs P, Bussel JB, Chong BH, Cines DB, Gernsheimer TB, Godeau $\mathrm{B}$, et al: International consensus report on the investigation and management of primary immune thrombocytopenia. Blood 115: 168-186, 2010.

12. Nomura S, Kurata Y, Tomiyama Y, Takubo T, Hasegawa M, Saigo K, Nishikawa M, Higasa S, Maeda Y and Hayashi K: Effects of bisphosphonate administration on the bone mass in immune thrombocytopenic purpura patients under treatment with steroids. Clin Appl Thromb Hemost 16: 622-627, 2010.

13. Sambrook PN, Kotowicz M, Nash P, Styles CB, Naganathan V, Henderson-Briffa KN, Eisman JA and Nicholson GC: Prevention and treatment of glucocorticoid-induced osteoporosis: A comparison of calcitriol, vitamin D plus calcium, and alendronate plus calcium. J Bone Miner Res 18: 919-924, 2003.

14. Cesareo R, Di Stasio E, Vescini F, Campagna G, Cianni R, Pasqualini V, Romitelli F, Grimaldi F, Manfrini S and Palermo A: Effects of alendronate and vitamin D in patients with normocalcemic primary hyperparathyroidism. Osteoporos Int 26: 1295-1302, 2015.

15. Li G, Lv CA, Tian L, Jin LJ and Zhao W: A retrospective study of alendronate for the treatment of ankylosing spondylitis. Medicine (Baltimore) 97: e10738, 2018.

16. Millonig G, Graziadei IW, Eichler D, Pfeiffer KP, Finkenstedt G, Muehllechner P, Koenigsrainer A, Margreiter R and Vogel W: Alendronate in combination with calcium and vitamin $D$ prevents bone loss after orthotopic liver transplantation: A prospective single-center study. Liver Transpl 11: 960-966, 2005.

17. Shapses SA, Kendler DL, Robson R, Hansen KE, Sherrell RM, Field MP, Woolf E, Berd Y, Mantz AM and Santora AC II: Effect of alendronate and vitamin $\mathrm{D}_{3}$ on fractional calcium absorption in a double-blind, randomized, placebo-controlled trial in postmenopausal osteoporotic women. J Bone Miner Res 26: 1836-1844, 2011. 
18. Siris ES, Adler R, Bilezikian J, Bolognese M, Dawson-Hughes B, Favus MJ, Harris ST, Jan de Beur SM, Khosla S, Lane NE, et al: The clinical diagnosis of osteoporosis: A position statement from the National Bone Health Alliance Working Group. Osteoporos Int 25: 1439-1443, 2014.

19. Hasegawa M, Nisikawa M, Nomura S, Takubo T, Suehiro A, Saigo K, Hayashi K, Tamaki S, Mizutani M, Uemura Y and Kurata Y; Kinki ITP Cooperative Study Group: Effect of glucocorticoids on bone mineral density in patients with idiopathic thrombocytopenic purpura. Rinsho Ketsueki 46: 121-126, 2005 (In Japanese).

20. Liao EY, Zhang ZL, Xia WB, Lin H, Cheng Q, Wang L, Hao YQ, Chen DC, Tang H, Peng YD, et al: Calcifediol (25-hydroxyvitamin D) improvement and calcium-phosphate metabolism of alendronate sodium/vitamin $\mathrm{D}_{3}$ combination in Chinese women with postmenopausal osteoporosis: A post hoc efficacy analysis and safety reappraisal. BMC Musculoskelet Disord 19: 210, 2018

21. Yang L, Tian JH,He ZY, Tang XL and Yang KH: A Meta-analysis of alendronate for the prevention and treatment of glucocorticoid-induced osteoporosis. Zhonghua Nei Ke Za Zhi 52: 838-843, 2013 (In Chinese).

22. D'Amelio P, Grimaldi A, Cristofaro MA, Ravazzoli M, Molinatti PA, Pescarmona GP and Isaia GC: Alendronate reduces osteoclast precursors in osteoporosis. Osteoporos Int 21: 1741-1750, 2010.
23. Bradaschia-Correa V, Moreira MM and Arana-Chavez VE: Reduced RANKL expression impedes osteoclast activation and tooth eruption in alendronate-treated rats. Cell Tissue Res 353: 79-86, 2013.

24. Martins CA, Leyhausen G, Volk J and Geurtsen W: Effects of alendronate on osteoclast formation and activity in vitro. J Endod 41: 45-49, 2015.

25. Zhang Q, Badell IR, Schwarz EM, Boulukos KE, Yao Z, Boyce BF and Xing L: Tumor necrosis factor prevents alendronate-induced osteoclast apoptosis in vivo by stimulating Bcl-xL expression through Ets-2. Arthritis Rheum 52: 2708-2718, 2005.

26. Siebelt M, Waarsing JH, Groen HC, Müller C, Koelewijn SJ, de Blois E, Verhaar JA, de Jong $\mathbf{M}$ and Weinans $\mathrm{H}$ : Inhibited osteoclastic bone resorption through alendronate treatment in rats reduces severe osteoarthritis progression. Bone 66: 163-170, 2014

27. Iseri K, Iyoda M, Watanabe M, Matsumoto K, Sanada D, Inoue T, Tachibana $S$ and Shibata T: The effects of denosumab and alendronate on glucocorticoid-induced osteoporosis in patients with glomerular disease: A randomized, controlled trial. PLoS One 13: e0193846, 2018.

28. Boyce AM, Kelly MH, Brillante BA, Kushner H, Wientroub S, Riminucci M, Bianco P, Robey PG and Collins MT: A randomized, double blind, placebo-controlled trial of alendronate treatment for fibrous dysplasia of bone. J Clin Endocrinol Metab 99: 4133-4140, 2014 